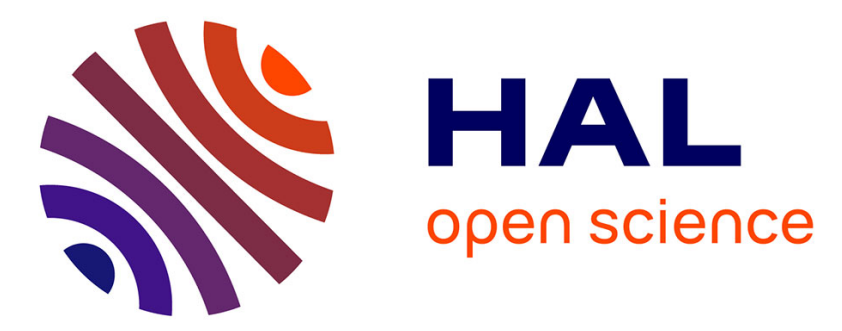

\title{
Optimizing the speed of single infrared-laser-induced thermocapillary flows micromanipulation by using design of experiments
}

Elvin Muñoz, Johan Quispe, Pierre Lambert, Aude Bolopion, Ronald Terrazas, Stéphane Régnier, Emir Vela

\section{To cite this version:}

Elvin Muñoz, Johan Quispe, Pierre Lambert, Aude Bolopion, Ronald Terrazas, et al.. Optimizing the speed of single infrared-laser-induced thermocapillary flows micromanipulation by using design of experiments. Journal of Micro-Bio Robotics, 2017, 12 (1 - 4), pp.65 - 72. hal-02963645

\section{HAL Id: hal-02963645 \\ https://hal.science/hal-02963645}

Submitted on 11 Oct 2020

HAL is a multi-disciplinary open access archive for the deposit and dissemination of scientific research documents, whether they are published or not. The documents may come from teaching and research institutions in France or abroad, or from public or private research centers.
L'archive ouverte pluridisciplinaire $\mathbf{H A L}$, est destinée au dépôt et à la diffusion de documents scientifiques de niveau recherche, publiés ou non, émanant des établissements d'enseignement et de recherche français ou étrangers, des laboratoires publics ou privés. 


\title{
Optimizing the Speed of Single Infrared-Laser-Induced Thermocapillary Flows Micromanipulation by using Design of Experiments
}

\author{
Elvin Muñoz* • Johan Quispe* • Pierre Lambert • Aude Bolopion • \\ Ronald Terrazas • Stéphane Régnier • Emir Vela ${ }^{\dagger}$
}

Received: date / Accepted: date

\begin{abstract}
Laser-induced thermocapillary convection flows tion process using a systematic method: Design of Exis a promising technique to manipulate micrometer size particles. Several parameters, such as the laser exposure time, the laser-particle distance, the particles' diameter and the water layer thickness can be used to control the particles' speed. This article deals with the study of the influence of the control parameters in the manipulaperiments (DoE). Additionally, a mathematical speed model of the manipulated particle as function of the mentioned parameters is proposed in order to enhance the manipulation accuracy and speed paving the way toward future works related to control strategies. Acidwashed glass beads ranging from 50 up to 150 microns were used for this purpose.
\end{abstract}

* These authors contributed equally to this work.

${ }^{\dagger}$ Corresponding author.

Elvin Muñoz and Johan Quispe

Department of Mechanical Engineering, Universidad de Ingeniería y Tecnología - UTEC, Jr. Medrano Silva 165,

Barranco, Lima 04, Perú

E-mail: elvin.mark.mv@gmail.com

E-mail: jequispenavarrete@gmail.com

Pierre Lambert and Ronald Terrazas

Bio-, Electro- And Mechanical Systems Department

(BEAMS), Ecole Polytechnique de Bruxelles, Université

Libre de Bruxelles, 1050 Bruxelles, Belgium

E-mail: pierre.lambert@ulb.ac.be

E-mail: rterraza@ulb.ac.be

Aude Bolopion

FEMTO-ST Institute, AS2M Department, Univ. Bourgogne

Franche-Comté, Univ. de Franche- Comté/CNRS/ENSMM,

24 rue Savary, 25000 Besançon, France

E-mail: aude.bolopion@femto-st.fr

Stéphane Régnier

Institut des Systèmes Intelligents et de Robotique (ISIR),

Pierre et Marie Curie University, 4 Place Jussieu, 75005

Paris, France

E-mail: stephane.regnier@upmc.fr

Emir Vela

Department of Mechanical Engineering, Universidad de Ingeniería y Tecnología - UTEC, Jr. Medrano Silva 165, Barranco, Lima 04, Perú

Department of Electrical and Computer Engineering, The

University of New Mexico, Albuquerque, New Mexico, USA

E-mail: evela@utec.edu.pe

Keywords Infrared Laser . Thermocapillary flows . Design of Experiments (DoE) · Micromanipulation

\section{Introduction}

Non contact actuation is now widely used to manipulate objects at the micrometer scale [1]. Compared to classical approaches that use mainly tips, grippers or pipettes $[2,3]$ it is based on force fields produced by remote sources. These remotely actuated systems can be classified into three categories depending on how the force field is used to interact with the object: (i) the force fields are used to control remotely actuated tools that interact with the object, (ii) the force fields directly control the objects or (iii) the force fields are used to modify the environment in which the object is placed. A few examples of each category are reviewed below.

Remotely controlled microtools have been developed to handle micrometer size objects. In [4] closed-loop control of untethered grippers driven by magnetic fields and automated pick-and-place of biological material on porcine tissue in an unstructured environment are demonstrated. Magnetic fields are also used in [5] to control microrobots fabricated from polymer (SU-8) with internal soft-magnetic posts having a maximum dimension of $50 \times 200 \times 600 \mu^{3}$. Indirect pushing of biological 
cells using an optically trapped tool is demonstrated in [6] and [7]. The use of tools to push the cells minimizes their exposure to the laser beam and the related generated heat.

The second category of remotely actuated systems consists in using the remote force fields to directly control the object of interest, without intermediate manipulator. Electric fields are widely used for that purpose, especially for in vitro cell analysis. As reviewed in [8] and [9] electric fields can induce electrophoresis (movement of charged particles in an electric field), or dielectrophoresis (motion of polarizable particles in a time variant electric field). Electric fields can also modify the cell structure via electroporation (formation of aqueous pores in the cell membrane) or electrofusion (fusion of cells). Closed loop trajectory control of artificial objects is demonstrated using dielectrophoresis [10,11], as well as controlled orientation [12]. In addition to electrical fields, magnetic ones can also be used for micromanipulation. Important forces can be applied using electromagnetic coils. However the object to be manipulated must have magnetic properties [13]. Optical tweezers are demonstrated in [14] and [15]. This technique shows promising results for the independent control of several objects simultaneously. It is commonly used to manipulate biological cells [16], or to analyze them. In [17] the authors use optical tweezers to get information about the mechanisms of chemoattractant gradient induction of cell migration. However it proves to be difficult to manipulate objects larger than several tens of micrometers using optical tweezers. Other techniques, such as acoustic waves can be considered to manoeuvre cells as detailed in [18].

In the third category, manipulation via actuated flows is another option. In this type of manipulation, fluid motion generated through different methods (i.e. electroosmosis, electrohydrodynamics, thermocapillary pumping [19]) is used to displace the desired object. Among the different mechanisms to generate flows, surface tension gradients are very promising because the surface effects are significant at the micro-scale. Flows generated by surface tension gradients are named as Marangoni convection flows. However, if the surface tension gradient is generated by a temperature gradient parallel to the surface, these flows are known as thermocapillary convection flows [20]. Moreover, there are some variations in the manner of heating and as a consequence, it produces different types of instabilities and patterns. Nevertheless, if a laser is used as heat source to produce the thermocapillary flows, toroidal convection cells patterns are very likely to occur. Ther- mocapillary flows have been already successfully used for micro-object manipulation. In [21], the authors used thermocapillary convection flows generated around a bubble heated by a light pattern which was confined on a closed chamber. The generated flow repelled the object from the bubble if it was floating and attracted the object if it was sunken. By using this technique, different sized particles were manipulated such as $60 \mu \mathrm{m}-$ radius glass beads and SU-8 triangular-shaped components of $360 \times 185 \times 100 \mu \mathrm{m}^{3}$. In [22] and [23], the authors generated the thermocapillary convection flows in a slightly different manner. A laser-light absorbent substrate is heated strongly enough to, first, create a bubble microrobot in the target illuminated position and, then, generate flows around those bubbles. In [24], the bottom of a water layer was heated using a laser to generate Marangoni convection flows. Using this principle, glass beads with diameters $31,62,92 \mu \mathrm{m}$ were displaced with speeds up to $2,3,4 \mathrm{~mm} / \mathrm{s}$ respectively inside a 450 $\mu \mathrm{m}$ water layer. Recently, [25,26] study laser-induced thermocapillary convection flows. The authors analyse the convection cell growth to manipulate micro-objects accurately [25]. They prove that it is possible to separate particles by controlling the motion of the desired particle with the convection cell without altering the position of other objects [26]. However, none of these works study in detail the influence of the control parameters on the particle's position and speed. So that thermocapillary flows can be used for controlled micromanipulation, this issue is of utmost importance.

This paper provides a systematic analysis of the most influential parameters namely: the laser exposure time, the distance between the laser and the manipulated particles, the particle's diameter and the water layer thickness. Design of Experiments (DoE) method is conducted to analyze the impact on the particle's speed. This work paves the way toward fully controlled micromanipulation via thermocapillary flows.

\section{Thermocapillary flows based micromanipulation}

\subsection{Thermocapillary flows physical principle}

Marangoni convection flow is a phenomenon which is generated by a surface tension gradient on a fluid-fluid interface. The surface tension $\sigma$ is a function of the pressure $P$ and the temperature $T$ on the interface as it is expressed in the following equation:

$d \sigma=\frac{\partial \sigma}{d P} d P+\frac{\partial \sigma}{\partial T} d T$ 
Fig. 1 Recirculating flows can move sedimented objects toward the Infra-Red Laser Beam (IRLB). The velocity profile shows a geometric relation between the Upper Subsurface Flows (USF) and the Lower Subsurface Flows (LSF) (USF/LSF = 1/2) [27]. This relation is kept even if there is a deformation of the interface caused by the relative viscosity on the interface (water-air in our case) $[28,29]$. (i) and (ii) are respectively the contribution of the dragging and the rolling movements to the particle's motion.

and if any of these physical parameters is altered, the surface tension will change. Moreover, if a fluid-fluid interface is only heated $\left(d \sigma=\frac{\partial \sigma}{\partial T} d T\right)$, it is more suitable to name this phenomenon as thermocapillary convection. Furthermore, if a laser is used in order to heat the interface we are referring to laser-induced thermocapillary flows. As it was mentioned, this phenomenon is initially produced on the interface. Due to the unbalanced shear stress produced on it, the fluid on the interface is pulled from the hot regions (low surface tension) to regions with higher values of surface tension. If we consider the heat source as a point, the phenomenon will become axisymmetric from this reference point. As a consequence of the mass conservation equation on the control volume, recirculation flows are generated and sedimented objects can be moved toward the laser beam center (see Figure 1). However, until this moment it is not established whether the particle is either dragged, rotated or both. A velocity model for thermocapillary flows was shown in Da Costa's work [27]. It derives the velocity field from Levich's work [30]. Additionally, an interesting result appears in this work: a geometric relation between the Upper Subsurface Flows (USF) and the Lower Subsurface Flows (LSF) was found. LSF appears from $z=0$ to $z=\frac{2 H}{3}$ and USF appears from $z=\frac{2 H}{3}$ to $z=H$. However, there is a local maximum for the velocity in $z=\frac{H}{3}$ and it can produce different effects on the particle's kinetics depending on its size and z-position.

\subsection{Pulsed based manipulation strategy}

In previous works $[25,26]$, the main outcome was the separation of particles. This task was achieved by characterizing the movement of a certain sedimented particle immersed into the fluid and by controlling the growth of the convection cell. These concepts brought into light a new micro manipulation technique. Selective manipulation of objects can also be performed by controlling the transient regime of single-IRLB-generated thermocapillary flows thus overcoming the toroidal shape of the flows; it means particles can be separated out of a group. In [31], it was shown the existence of stationary convection cells. However, at the micro-scale due to the prevalence of the surface forces, these convection cells, which are recirculating flows, undergo a transient regime. This transitory recirculating flow can be ex- ploited to move particles in a fast and accurate manner. These transitory convection cells are generated by heating the interface with an Infrared Laser Beam (IRLB) for a brief duration (IRLB pulses). IRLB pulses are thus a promising solution to move micro-objects by thermocapillary flows based micromanipulation. This strategy will be used in the rest of this paper.

To perform an efficient manipulation based on IRLB pulses the duration of the laser pulse should be tuned. The distance between the IRLB and the particle of interest is also an important parameter. When the convection cell is evolving the kinetics varies with respect to the position inside the convection cell. The force exerted by the flows on the particle is going to change rapidly. There is thus an optimal laser-particle distance in order to drag the particle strongly enough for a fixed pulse time.

\subsection{Experimental Setup}

The experimental setup is shown in Figure 2. The main components of the setup are labeled in the picture. The infrared laser ( $\lambda=1470 \mathrm{~nm}$, Laserglow) is used as the heat source. The output beam from the laser is reflected by two silver-coated mirrors ( $95 \%$ of reflectance on the IR range) mounted on a galvanometer scanner (Thorlabs GVS002). These mirrors, which are each attached to a high-precision and stable servo motors can be rotated from $-12.5^{\circ}$ to $12.5^{\circ}$ from their neutral state. The reflected beam takes different output angles which makes the beam hit the Petri-dish in different XY positions. This beam is then magnify $(1.5 \times)$ by a Keplerian arrange of lenses. The magnified beam is reflected by a dichroic mirror and directed toward a microscope objective $(4 \times)$ which focalizes the laser at the bottom of the Petri-dish. This focalized laser beam heats the film of water inside the Petri-dish which generates thermocapillary convection flows. These flows are used in this work for the displacement of immersed particles. The dichroic mirror reflects all the infrared laser radiation but it lets the visible light pass through it, so a high speed camera (DALSA Genie HM1400) underneath can record images of what is occurring inside the film of water in the Petri-dish. The ON and OFF switching of the infrared laser to produce the pulses as well as the an- 
Fig. 3 Parameters involved in the manipulation strategy. $H$ : water layer thickness, $D$ : particle's diameter, $L$ : laser-particle distance, $\tau$ : pulse duration.

Fig. 4 Each curve represents the evolution of the particle's speed during the manipulation for different combination of water layer thickness (H), particle's diameter (D), laserparticle distance $(\mathrm{L})$, pulse duration $(\tau)$.

gular position of the mirrors of the galvanometer scanner are electronically controlled with a data acquisition card (NIDAQ-USB 6211).

2.4 Influence of the geometrical and control parameters on the manipulation speed

The manipulation strategy has been defined in Sec. 2.2. It consists in heating a water layer of thickness $H$ using IRLB pulses. The laser is switched on during a time $\tau$. It heats the water at a distance $L$ from a particle of diameter $D$ (see Figure 3). The manipulated particles were acid-washed glass beads ranging from 50 up to 150 microns, from Sigma-Aldrich. This work aims to find the optimal values of these parameters in order to achieve the shortest period of time for a particle to reach a desired position.

The influence of these parameters on the particle's speed is shown experimentally in Figure 4. This figure presents the particle's speed evolution for three different sets of parameters. The saw-shaped curve in each graphics is due to the pulse technique used in this work. Furthermore, it is clearly noticeable that the maximum speed reached in each case varies from one set of parameters to another. From these experiments it is hard to say which parameter influence the most the velocity of the particle. To do so a design of experiment is proposed in the next section.

\section{Design of Experiments (DoE)}

\subsection{Brief Introduction}

The design of experiments, or DoE for short, is an important tool to measure how the variations of some so-called factors affect the output of a process also called response [32]. The values of these factors are varied systematically in a certain number of levels and the response for each combination is measured. Analyzing these responses makes possible to determine which factor impacts the most. Moreover a local linearapproximation, or a higher order approximation if required, can be done, giving a mathematical formula of the evolution of the response of the system with respect to the variation of the factors valid within the analyzed range which can served for future applications. Thus, in this work, it was proposed to use this method to analyze the contribution of each factor to the particle's speed. Specifically, a two-level full factorial design is performed.

\subsection{Two-level full factorial design}

A full factorial design means that all the combinations of all the levels for each factor are analyzed. More specifically, a two-level full factorial design involves just 2 levels: a minimum level and a maximum one for each factor. So if $\mathrm{k}$ represents the number of factors involved in the process then the number of runs would be $2^{k}$. Plus these runs, some repetitive runs in the central point are added in order to see the lack-of-fit and depending on its significance, the experiments can be redesigned for a higher order approximation.

Since we have 4 factors $(H, D, L$ and $\tau)$, we need $2^{4}=16$ runs plus 4 central points runs thus giving a total of 20 runs as shown in Table 1 . The levels, minimum and maximum, are given due to the limitations of our system. For the water layer thickness $(H)$, the minimum value that it can take is around $200 \mu \mathrm{m}$, since below this value the Petri-dish is not filled homogeneously, while the maximum value is about $300 \mu \mathrm{m}$ since higher values do not allowed a proper heating of the film of water. In the case of the particles' diameter $(D)$, the minimum value, $50 \mu \mathrm{m}$, and the maximum value, $150 \mu \mathrm{m}$, were taken as such since they were the most frequent sizes available in our samples. The range for the laser-particle distance $(L)$ was chosen taking into account the size of our workspace $(2 \mathrm{~mm} \times 2 \mathrm{~mm})$ and the maximum average particle's diameter $(150 \mu \mathrm{m})$ found in our samples. Thus the minimum value of $L$ was $225 \mu \mathrm{m}$, which is 1.5 times $150 \mu \mathrm{m}$, and the maximum value of $L$ was $325 \mu \mathrm{m}$, which is $\sim 0.15$ times $2 \mathrm{~mm}$. For the pulse duration, the levels were found experimentally by trial and error testing if the particle moves or not for the chosen range of $L$. The central point for each level is given by adding the extreme values and dividing it by 2. A summary of the values of the levels for each factor is presented in Table 2 . 
Fig. 2 Experimental setup presenting the main optical and electronic components. (a) Front view. (b) Top view. (c) Image acquired by the camera.

Table 1 Experiment table with each run where "-1" represents the minimum value, "0" represents the central value and " +1 " represents the maximum value

\begin{tabular}{lllll}
\hline $\mathrm{H}$ & $\mathrm{D}$ & $\mathrm{L}$ & $\tau$ & Response \\
\hline-1 & -1 & -1 & -1 & $R_{1}$ \\
-1 & -1 & -1 & +1 & $R_{2}$ \\
-1 & -1 & +1 & -1 & $R_{3}$ \\
-1 & -1 & +1 & +1 & $R_{4}$ \\
-1 & +1 & -1 & -1 & $R_{5}$ \\
-1 & +1 & -1 & +1 & $R_{6}$ \\
-1 & +1 & +1 & -1 & $R_{7}$ \\
-1 & +1 & +1 & +1 & $R_{8}$ \\
+1 & -1 & -1 & -1 & $R_{9}$ \\
+1 & -1 & -1 & +1 & $R_{10}$ \\
+1 & -1 & +1 & -1 & $R_{11}$ \\
+1 & -1 & +1 & +1 & $R_{12}$ \\
+1 & +1 & -1 & -1 & $R_{13}$ \\
+1 & +1 & -1 & +1 & $R_{14}$ \\
+1 & +1 & +1 & -1 & $R_{15}$ \\
+1 & +1 & +1 & +1 & $R_{16}$ \\
0 & 0 & 0 & 0 & $R_{17}$ \\
0 & 0 & 0 & 0 & $R_{18}$ \\
0 & 0 & 0 & 0 & $R_{19}$ \\
0 & 0 & 0 & 0 & $R_{20}$ \\
\hline
\end{tabular}

Table 2 Levels for each factor

\begin{tabular}{llll}
\hline Factor & Min. (-1) & Central (0) & Max. (+1) \\
\hline $\mathrm{H}$ & $200 \mu \mathrm{m}$ & $250 \mu \mathrm{m}$ & $300 \mu \mathrm{m}$ \\
$\mathrm{D}$ & $50 \mu \mathrm{m}$ & $100 \mu \mathrm{m}$ & $150 \mu \mathrm{m}$ \\
$\mathrm{L}$ & $225 \mu \mathrm{m}$ & $275 \mu \mathrm{m}$ & $325 \mu \mathrm{m}$ \\
$\tau$ & $100 \mathrm{~ms}$ & $160 \mathrm{~ms}$ & $220 \mathrm{~ms}$ \\
\hline
\end{tabular}

\section{Experimental Results}

\subsection{Analyzing the influence of all the parameters}

Experiments are conducted for each run given in Table 1. Each run is repeated 3 times to see how repeatable our data are. In order to compare the contribution of each parameter and their interactions to the average speed, the data are standardized using the Z-score method [33]. Since we are comparing the influence of parameters with different orders of magnitudes and units of measure such as $H[\mu m]$, which is in the order of $10^{2}$, and $H * L\left[\mu m^{2}\right]$, which is in the order of $10^{4}$, it is impossible to analyze the given data as such. The Z-score method allows to compare their influence by transforming the data set from its original form to a standardized form. This transformation is done by the following equation:

$X^{*}=\frac{X-\bar{X}}{\sigma}$ where $X^{*}$ is the standardized data, $X$ is the original data, $\bar{X}$ is the mean value of the data set and $\sigma$ is the standard deviation. The standardized values are a measurement of how much each data is away from the mean value, but expressed in terms of sigma, therefore, the order of magnitudes as well as the units of measurements of the data does not matter.

A linear regression is applied to the original data and standardized data using the following model:

$$
\begin{aligned}
\text { Speed } & =a_{0}+a_{1} H+a_{2} D+a_{3} L+a_{4} \tau+a_{5} H * D+a_{6} H * L \\
& +a_{7} H * \tau+a_{8} D * L+a_{9} D * \tau+a_{10} L * \tau
\end{aligned}
$$

where the coefficients of each term $a_{i}$ are going to be determined by a linear regression.

The standardized coefficients obtained through this method are shown in Figure 5 and a brief summary of the linear regression's analysis is presented in Table 3. As it can be observed in the table, the p-values of some parameters and interactions are much greater than 0.05 which means that they are not contributing at all to the speed response [32]. All the apparent variations on the response by varying these parameters can be considered as pure noise and do not represent a true effect. On the other hand, the parameter $H$ and $L$ and their interactions have p-values smaller than 0.05 which means that they indeed represent a true effect on the response. Moreover, the standardized coefficients (given in Figure 5) show that the major contributors to the speed are exactly the same terms that have the small p-values $(<0.05)$. The model could thus be simplified as it will be discussed in Sec. 4.2.

From this, it is deduced that neither $D$ nor $\tau$ are factors that affect the particle's speed. However the physical explanation of thermocapillary manipulation presented in Sec. 2.1 highlights that there is undoubtedly an influence of the ratio between the particle's diameter and the water layer thickness on the particle's speed. A separate study is thus carried out to find this effect (Sec. 4.3).

Concerning $\tau$, this factor indeed does not affect the particle's speed. For a given $L$, there is a given threshold time $\left(\tau_{0}\right)$ for which the particle starts moving [25]. For the selection of the levels the values of $\tau$ were taken so that the particle moves. In other words, all the values of $\tau$ are such that $\tau=\tau_{0}+\epsilon$ where $\epsilon>0$. Therefore, slight variations of $\epsilon$ does not contribute at all to the particles' speed [24]. 
Fig. 5 Standardized coefficients obtained from the linear regression presented as bars. Model: Speed $=a_{0}+a_{1} H+a_{2} D+$ $a_{3} L+a_{4} \tau+a_{5} H * D+a_{6} H * L+a_{7} H * \tau+a_{8} D * L+a_{9} D *$ $\tau+a_{10} L * \tau$. At this step all the interactions are considered.

Fig. 6 Standardized coefficients obtained from the linear regression presented as bars. Model: Speed $=a_{0}+a_{1} H+a_{2} L+$ $a_{3} H * L$. Just $\mathrm{H}, \mathrm{L}$ and the their interaction are consider in this model.

Fig. 7 Surface Speed Response along with the experimental data. Speed $=2 \times 10^{4}-64.1 H-55.5 L+0.2 H * L$.

Fig. 8 Variation of the particle's speed according to the ratio of the particle's diameter (D) and the water layer thickness (H). $L=275 \mu \mathrm{m}$ and $\tau=160 \mathrm{~ms}$.

\subsection{Redesigning the model: $\operatorname{Speed}(H, L)$}

According to the previous section $H$ and $L$ are the only factors that affect the particle's speed. Equation (3) can thus be simplified as:

Speed $=a_{0}+a_{1} H+a_{2} L+a_{3} H * L$

where $a_{i}$ are the coefficients to be obtained by the linear regression. The same procedure as previously is carried out leading to the standardized coefficients obtained by linear regression shown in Figure 6 . Table 4 shows a summary of the analysis of the linear regression. As it can be observed in the table, the p-values of all the terms in this model are less than 0.05 which means that all of them indeed have a true effect on the response. Moreover, as it can be seen from the impact of each factor in the speed, $H$ has a negative contribution to the speed as well as $L$ which means that in order to increase the speed, both parameters have to be decreased (the water layer thickness should be small, and the laser should heat the water close to the particle). Furthermore, the F-statistic value is greater than 5, and the upper tail probability of the F-distribution corresponding to the value of F-statistic is also much smaller than 0.05 , both values indicate that the lack-of-fit is nonsignificant which means that a linear approximation is enough to model the particle's speed within the analyzed range.

A 3D representation of the surface speed response is shown in Figure 7 which is plotted using the model: Speed $=2 \times 10^{4}-64.1 H-55.5 L+0.2 H * L$. It is also shown the experimental data $(H, L, S p e e d)$ as scatter points. It is observed that these experimental points do not differ pretty much from the surface which means that the linear model is an enough approximation to model the experimental data.
Table 3 Summary of the analysis of the linear regression for the model: Speed $=a_{0}+a_{1} H+a_{2} D+a_{3} L+a_{4} \tau+a_{5} H * D+$ $a_{6} H * L+a_{7} H * \tau+a_{8} D * L+a_{9} D * \tau+a_{10} L * \tau$. F-statistic $=5, \operatorname{Prob}($ F-statistic $)=0.013$.

\begin{tabular}{lllll}
\hline & Coefficients & $\begin{array}{c}\text { Standardized } \\
\text { coefficients }\end{array}$ & $\begin{array}{c}\text { Standard } \\
\text { deviation }\end{array}$ & p-value \\
\hline Intercept & $2.03 \times 10^{4}$ & $4.441 \times 10^{-16}$ & 4309 & 0.001 \\
H & -68.5 & -0.54 & 14.7 & 0.001 \\
D & -19.9 & 0.069 & 17.9 & 0.295 \\
$\mathrm{~L}$ & -55.5 & -0.33 & 13.7 & 0.003 \\
$\tau$ & 6.7 & 0.23 & 14.6 & 0.655 \\
$\mathrm{H}^{*} \mathrm{D}$ & 0.07 & 0.2 & 0.045 & 0.131 \\
$\mathrm{H}^{*} \mathrm{~L}$ & 0.2 & 0.52 & 0.045 & 0.002 \\
$\mathrm{H}^{*} \tau$ & -0.02 & -0.061 & 0.038 & 0.617 \\
$\mathrm{D}^{*} \mathrm{~L}$ & $0.6 \times 10^{-3}$ & 0.002 & 0.045 & 0.99 \\
$\mathrm{D}^{*} \tau$ & 0.01 & 0.04 & 0.038 & 0.721 \\
$\mathrm{~L}^{*} \tau$ & $-6.6 \times 10^{-6}$ & $-2.1 \times 10^{-5}$ & 0.038 & 1 \\
\hline
\end{tabular}

Table 4 Summary of the analysis of the linear regression for the model: Speed $=a_{0}+a_{1} H+a_{2} L+a_{3} H * L$. F-statistic $=$ 14.7, Prob(F-statistic) $=7.3 \times 10^{-5}$

\begin{tabular}{lllll}
\hline & Coefficients & $\begin{array}{c}\text { Standardized } \\
\text { coefficients }\end{array}$ & $\begin{array}{c}\text { Standard } \\
\text { deviation }\end{array}$ & p-value \\
\hline Intercept & $2 \times 10^{4}$ & $4.441 \times 10^{-16}$ & 3157 & 0.0001 \\
H & -64.1 & -0.54 & 12.4 & 0.0001 \\
L & -55.5 & -0.32 & 11.3 & 0.0001 \\
$\mathrm{H}^{*} \mathrm{~L}$ & 0.2 & 0.52 & 0.04 & 0.0001 \\
\hline
\end{tabular}

4.3 Analyzing the influence of the ratio: $\frac{D}{H}$

As mentioned before, the physical analysis of the manipulation technique underlines the influence of the ratio between the particle's diameter and the water layer thickness on the particles' speed. A specific attention is given to this ratio in this section. To do this, the other two parameters ( $L$ and $\tau$ ) are fixed at their central values and speed tests are conducted for several ratios $D / H$. The results are shown in Figure 8. The particle's speed is indeed affected; nevertheless, the variations on the particle's speed are less than $1000 \mu \mathrm{m} / \mathrm{s}$. This maximum variation is the same order of magnitude than the noise. This is the reason why the influence of the diameter was unnoticed in the model section 4.1 as it was confused with noise. Moreover, Figure 8 indicates that there are 2 peaks that are located approximately at the ratios: $\frac{1}{3}$ and $\frac{2}{3}$. The local maximum at those ratios are explained due to the shape of the flows' velocity profile (see Section 2). As it was shown in Figure 1, the ratio between the lower subsurface flows and the thickness of the water layer is $\frac{2}{3}$ and due to the parabolic shape of the flows' velocity profile, the inflexion point of the lower subsurface flows occurs at $\frac{1}{3}$ of the water layer thickness. Both values correspond to the two peaks found in the graphics. These results are in good agreement with the underlying physical phenomena. 


\section{Conclusion}

This paper focuses on the determination of the most influential control parameters for thermocapillary pulse based manipulation. From the four parameters identified at first, a systematic analysis involving DoE highlights that just two of them significantly contribute to the particle's speed: the laser particle distance $(L)$ and the water layer thickness $(H)$. The water layer thickness $H$ is indeed the one that is the most influential variable. Within the analyzed range, it was determined that in order to maximize the speed, both parameters have to take their minimum values in the explored range. This means that the water layer thickness should be small, and the laser should be closed to the object. Moreover, the speeds, obtained experimentally, presents a relatively high level of dispersion which is due to the uncertainty in the z-position of the manipulated beads. Even though this might cause a problem in the modeling, the multiple repetitions of the experiments showed that the trend remains.

Furthermore, a separate study of whether the ratio $\frac{D}{H}$ influence or not on the particle's speed was done. The results showed that indeed, there is a dependency of the speed with the ratio $\frac{D}{H}$ and it reveals the existence of two peaks for which the speed is locally maximized.

This work proposes a mathematical approximation of the velocity of the particle using DoE. This model is a first step toward future control strategies for pulse based thermocapillary manipulation.

Acknowledgements This work was supported by the Programa Nacional de Innovación para la Competitividad y Productividad (FINCyT), Ministerio de la Producción, Perú, with grant \#394-PNICP-PIBA-2014.

\section{References}

1. M. Gauthier, N. Andreff, E. Dombre, Intracorporeal Robotics. From Milliscale towards Nanoscale, wiley edn. (2014)

2. H. Xie, C.D. Onal, S. Régnier, M. Sitti, Atomic Force Microscopy Based Nanorobotics, Springer Tracts in Advanced Robotics, vol. 71, modelling, simulation, setup building and experiments series edn. (2011)

3. P. Bøggild, Micro and Nano Techniques for the Handling of Biological Samples p. 141 (2011)

4. F. Ongaro, S. Scheggi, C. Yoon, F. van den Brink, S.H. Oh, D.H. Gracias, S. Misra, Journal of Micro-Bio Robotics pp. 1-8 (2016)

5. R.S. Pieters, H.W. Tung, B.J. Nelson, Microrobots for Active Object Manipulation (Springer International Publishing, 2017), pp. 61-72

6. A. Thakur, S. Chowdhury, P. Svec, C. Wang, W. Losert, S.K. Gupta, The International Journal of Robotics Research p. 0278364914523690 (2014)
7. F. Arai, H. Maruyama, T. Sakami, A. Ichikawa, T. Fukuda, IEEE/ASME transactions on mechatronics 8(1), 3 (2003)

8. J. Voldman, Annual Review of Biomedical Engineering $\mathbf{8}, 425(2006)$

9. J. Čemažar, D. Miklavčič, T. Kotnik, J. Microelectron. Electron. Compon. Mater 43, 143 (2013)

10. M. Kharboutly, M. Gauthier, in IEEE International Conference on Robotics and Automation (2013), pp. $1438-1443$

11. J. Zemanek, J. Drs, Z. Hurak, in IEEE/ASME International Conference on Advanced Intelligent Mechatronics (2014), pp. 19-25

12. C. Jiang, J.K. Mills, IEEE/ASME Transactions on Mechatronics 20(5), 2350 (2015)

13. M. Dkhil, M. Kharboutly, A. Bolopion, S. Régnier, M. Gauthier, IEEE Transactions on Automation Science and Engineering pp. 1-13 (2015)

14. K. Onda, F. Arai, in IEEE International Conference on Robotics and Automation (2012), pp. 1069 -1074

15. X. Li, H. Yang, J. Wang, D. Sun, Automatica 55, 279 (2015)

16. H. Chen, D. Sun, IEEE Transactions on Robotics 28(5), 1069 (2012)

17. H. Yang, X. Gou, Y. Wang, T.M. Fahmy, A.Y.H. Leung, J. Lu, D. Sun, Biophysical journal 108(7), 1645 (2015)

18. S.C.S. Lin, X. Mao, T.J. Huang, Lab on a chip 12(16), 2766 (2012)

19. M.A. Burns, C.H. Mastrangelo, T.S. Sammarco, F.P. Man, J.R. Webster, B.N. Johnsons, B. Foerster, D. Jones, Y. Fields, A.R. Kaiser, D.T. Burke, in Proceedings of the National Academy of Sciences of the United States of America, 93(11), vol. 93 (1996), vol. 93, pp. 5556-5561

20. M.F. Schatz, G.P. Neitzel, Annual Review of Fluid Mechanics 33(1), 93 (2001). DOI 10.1146/annurev.fluid.33.1.93

21. W. Hu, K.S. Ishii, A.T. Ohta, Applied Physics Letters 99(9), 094103 (2011). DOI 10.1063/1.3631662

22. W. Hu, Q. Fan, A.T. Ohta, Lab Chip 13(12), 2285 (2013). DOI 10.1039/C3LC50389E

23. W. Hu, Q. Fan, A.T. Ohta, Robotics and Biomimetics 1(1), 1 (2014). DOI 10.1186/s40638-014-0014-3

24. E. Vela, Non-contact microscale manipulation using laser-induced convection flows. Ph.D. thesis, Universite Pierre et Marie Currie (2010)

25. J. Quispe, E. Muñoz, E. Vela, Applied Physics Letters 109(12), 124102 (2016)

26. J.E. Quispe, J.C. Inga, E.M. Muñoz, S. Régnier, E. Vela, in Manipulation, Automation and Robotics at Small Scales (MARSS), International Conference on (IEEE, 2016), pp. 1-6

27. G. Da Costa, Applied optics 32(12), 2143 (1993)

28. A. Miniewicz, S. Bartkiewicz, H. Orlikowska, K. Dradrach, Scientific Reports 6 (2016)

29. H. Chraïbi, J.P. Delville, Physics of Fluids (1994-present) 24(3), 032102 (2012)

30. V. Levich, V. Krylov, Annual Review of Fluid Mechanics 1(1), 293 (1969)

31. S. Shin, I. Jacobi, H.A. Stone, EPL (Europhysics Letters) 113(2), 24002 (2016)

32. D.C. Montgomery, Design and Analysis of Experiments, 8th edn. (2012)

33. D.C. Shirley Dowdy, Stanley Wearden, Statistics for Research, 3rd edn. (2004) 